\title{
Machtverhältnisse und Wortspiele. Yōko Tawada und Plinius der Jüngere
}

\section{Yōko Tawada: Von der Muttersprache zur Sprachmutter (1996)}

Dass Machtverhältnisse im Schreib- und Kommunikationsprozess des literarischen Kunstbetriebs eine wichtige Rolle spielen, ist so alt wie die Literatur selbst. Mitunter begegnen sie an erwarteten, mitunter an überraschenden Stellen. In ihrem Essay Von der Muttersprache zur Sprachmutter legt Yōko Tawada einen Aspekt von Macht und Machtmetaphern frei, der erst im Licht von Mehrsprachigkeit und Translation klar hervortritt:

\begin{abstract}
Das ist der deutsche Animismus, dachte ich mir. Zuerst war ich nicht sicher, ob die Frau ihre Wut scherzhaft übertrieb oder ob sie wirklich so wütend war, wie sie aussah. Denn es war für mich nicht vorstellbar, so ein starkes Gefühl für einen so kleinen Gegenstand empfinden zu können. Ich bin zum Beispiel noch nie in meinem Leben über mein Schreibzeug wütend geworden. Die Frau schien aber - soweit ich es beurteilen konnte - ihre Worte nicht als Scherz gemeint zu haben. Mit einem ernsthaften Gesicht warf sie den Bleistift in den Papierkorb und nahm einen neuen in die Hand. Der Bleistift, der in ihrem Papierkorb lag, kam mir plötzlich merkwürdig lebendig vor.

Das war die deutsche Sprache, die der für mich fremden Beziehung zwischen diesem Bleistift und der Frau zugrunde lag. Der Bleistift hatte in dieser Sprache die Möglichkeit, der Frau Widerstand zu leisten. Die Frau konnte ihrerseits über ihn schimpfen, um ihn wieder in ihre Macht zu bekommen. Ihre Macht bestand darin, dass sie über den Bleistift reden konnte, während der Bleistift stumm war. (1996: 10)
\end{abstract}

Die metaphorische Verdinglichung der im Schreibprozess gegebenen Prozesse von Widerständigkeit und Überwindung im Objekt des Bleistifts, ist, wie Tawada im Folgenden ausführt, in ihrer Muttersprache von vornherein unmöglich, weil ,Bleistift' dort wie andere Worte auch kein grammatisches Geschlecht annehmen kann („Grammatikalisch ist im Japanischen nicht einmal der Mann männlich“, 1996: 11). Tawadas Anmerkungen zum ,deutschen Bleistift-Animismus‘ laden dazu ein, nach literarischen Machtverhältnissen an unerwarteten Orten zu suchen. Ein solcher findet sich im Briefwechsel des antiken Schriftstellers und Senatsaristokraten Plinius des Jüngeren (61-115 n. Chr.) mit Kaiser Trajan (53-117 n. Chr.). 


\section{Eco, Plinius und Trajan}

In einem Beitrag über Plinius den Jüngeren unter dem Titel How to build fame schließt Umberto Eco seine semiotische Analyse mit der folgenden Überlegung zur Funktion des ,idealen Lesers':

Fortunately every text is always, more or less consciously, for two kinds of Model Reader. The first is supposed to cooperate in actualizing the content of the text; the second is supposed to be able to describe - and enjoy - the way in which the first Model Reader has been textually produced. (1985: 302)

Jüngst ist Plinius’ zehnbändige Briefsammlung, nicht zuletzt im Gefolge Ecos, einer Vielzahl von Studien unterworfen worden, in denen sich ein wachsendes Interesse an Aspekten der literarischen Qualität abzeichnet. ${ }^{1}$ In Bezug auf die ersten neun Bücher der Sammlung dürfen wir annehmen, dass Auswahl, Zusammenstellung und Edition auf Plinius selbst zurückgehen. Sie umfassen insgesamt rund 247 Briefe, die sich über einen Zeitraum von mehr als zehn Jahren erstrecken (96-109 n. Chr.; vgl. Gibson und Morello 2012: 266). Dieses Korpus weist eine Reihe von Besonderheiten auf, so enthält sie nur die Briefe von Plinius' eigener Hand und lässt die Briefe der jeweils anderen Korrespondenzseite aus. Zur Gruppe der Adressaten zählt eine nicht unbedeutende Anzahl von Mitgliedern der senatorischen Elite seiner Zeit, darunter etwa der berühmte Historiker Publius Cornelius Tacitus (ca. 58-120 n. Chr.; vgl. Eco 1985; Gibson und Morello 2012: 161-168). ${ }^{2}$ Die Briefe bieten ein breites Themenspektrum, das von Einladungen über Empfehlungsschreiben zur literarischen Beschreibung von Villen und der Großkatastrophe des ersten Jahrhunderts, dem Ausbruch des Vesuvs im August 79 n. Chr., reicht, letzteres ein Ereignis, an dem auch Plinius' hochvermögender Onkel, Plinius der Ältere, den Tod fand. Sherwin-White hat acht typische Themenfelder identifiziert: zeitgenössische Politik, Charakterskizzen, Netzwerk und Patronage, moralische Ermahnungen und Ermunterungen, häusliche Angelegenheiten, Literatur, Ortsbeschreibungen (laus locorum) und soziale Höflichkeit (1966: 43-44; vgl. Gibson und Morello 2012: 293-307). Das zehnte Buch, worum es im Folgenden vor allem gehen soll, ist in mancherlei Hinsicht anders.

1 Vgl. zum Beispiel Marchesi (2008: XI): „Pliny’s epistles are neither a treatise nor a novel, nor even a dialogue in the classical sense; however, they are also far from being a chaotic assemblage of casually collected fragments“, Barchiesi (2005: 332), Ludolph (1997), Henderson (2002), Gibson und Morello (2012: 234-264), Marchesi (2015).

2 Zu den Adressaten s. Syme (1985), Sherwin-White (1966: 65-69), Birley (2000: 17-21), Gibson und Morello (2012: 136-168, 274-292). 
Hier finden sich beide Seiten der Korrespondenz, und Plinius' Briefpartner ist kein geringerer als der römische Kaiser Trajan selbst. Ungewiss ist, ob Plinius selbst eine Ausgabe auch dieser Briefe geplant hat oder ob die überlieferte Fassung erst nach seinem Tod von anderer Seite besorgt worden ist. Das Buch enthält insgesamt 121 Briefe, die sich in zwei Gruppen gliedern lassen: die erste enthält die Korrespondenz der Jahre 98 bis 102 n. Chr., die übrigen 107 Briefe sind ungefähr ein Jahrzehnt später verfasst worden und stammen aus der Periode, in der Plinius als kaiserlicher Statthalter der Provinz Bithynia et Pontus tätig war (heutige Nordwesttürkei, 109/112 n. Chr.). Themen sind Verwaltungsangelegenheiten, in denen Plinius in seiner öffentlichen Funktion als Statthalter verschiedene Entscheidungen treffen musste, die eine Konsultation des Kaisers in Rom erforderten. ${ }^{3}$

\section{Literarische Techniken in Plinius' Kaiser- korrespondenz}

Im Folgenden möchte ich die These aufstellen, dass Plinius in seiner Korrespondenz mit Kaiser Trajan teilweise dieselben literarischen Techniken anwendet, die jüngst vor allem in Bezug auf die Bücher eins bis neun diskutiert worden sind. ${ }^{4}$ Damit folge ich einem Ansatz, der generell eine engere Verbindung zwischen den ersten neun und dem zehnten Buch postuliert (Stadter 2006; Noreña 2007; Gibson und Morello 2012: 251-253; Woolf 2015). Unter diesen Techniken des zehnten Buches finden wir auch eine Art von Wortspiel, die man in einer offiziellen Korrespondenz zwischen Kaiser und Statthalter zunächst vielleicht nicht erwarten würde. Als Untersuchungsgegenstand für Wortspiele erscheint dieses Textcorpus gerade deshalb besonders reizvoll, weil es an der Schnittstelle zwischen Alltagskommunikation und Literatur steht (Zirker und Winter-Froemel 2015b: 1). ${ }^{5}$ Denn dass die Briefe über ihren unmittelbaren Alltagszweck hinaus

3 Einzelkommentare zu Buch 10: Williams (1990), Bracci (2011); das Buch ist auch berücksichtigt in dem Gesamtkommentar von Sherwin-White (1966). S. weiter Noreña (2007), Woolf (2015), Millar (2016a, 2016b), zur Provinz Bithynia et Pontus s. Woolf (2016).

4 Die hier behandelten Techniken sind gleichermaßen in Rhetorik und Literatur verankert. Im Folgenden wird etwas vereinfachend die literarische Seite betont, da die Briefe in ihrer vorliegenden Fassung einen Buchcharakter aufweisen, wie er auch sonst für den literarischen Kunstbrief der Antike typisch ist.

$5 \mathrm{Zu}$ Wortspielen in der lateinischen und griechischen Literatur des Altertums s. Ahl (1985), O’Hara (1996), Winter-Froemel (2009: 1433-1435), Mitsis und Ziogas (2016). 
auch einen literarischen Charakter aufweisen, wird an vielen Details deutlich, nicht zuletzt und für die hier behandelte Technik des Wortspiels von besonderer Relevanz an den intertextuellen Bezugnahmen einzelner Briefe und Briefsequenzen untereinander (Sherwin-White 1966: 533-535).

Bevor wir auf Plinius' Wortspielkunst näher eingehen, lohnt sich ein kurzer Blick auf die bisherige Bewertung seiner Rolle als Provinzstatthalter. Hier überwiegt eine Interpretationsrichtung, die in Plinius vor allem eine hochgradig unsichere und entscheidungsschwache Person sieht, die in allen wichtigen und auch weniger wichtigen Angelegenheiten Vorgaben aus Rom erbittet. Eine typische Stimme für diese Richtung ist Soverini (1989: 553), der Plinius als „un funzionario imprigionato in una completa dipendenza dalle direttive imperiali“ charakterisiert. Ähnlich urteilt auch Ludolph (1997: 53-54):

Fast scheint es erstaunlich, wie denn ein so desinteressierter Mann überhaupt eine doch beachtliche öffentliche Karriere machen konnte. Buch X gibt die Antwort auf diese Frage, denn in ihm erscheint Plinius als ein äußerst gewissenhafter, ja mitunter pedantischer Verwaltungsbeamter. [...] Mit Plinius' Pedanterie geht eine eklatante Entscheidungsschwäche einher - auch das ganz im Gegensatz zu den literarischen Briefen. Erscheint er dort als ,wise counsellor', so ist er im Buch X selbst derjenige, der ständig um Rat nachfragen muß. ${ }^{6}$

Eine solche Insekurität in der Amtsführung wird beispielhaft deutlich in Briefen wie 10.68-69, in denen Plinius beim Kaiser anfragt, wie er mit dem Wunsch der Lokalbevölkerung nach Umbettung ihrer Verstorbenen umgehen solle:

\subsection{PLINIUS TRAIANO IMPERATORI}

Petentibus quibusdam, ut sibi reliquias suorum aut propter iniuriam vetustatis aut propter fluminis incursum aliaque his similia quocumque secundum exemplum proconsulum transferre permitterem, quia sciebam in urbe nostra ex eius modi causa collegium pontificum adiri solere, te, domine, maximum pontificem consulendum putavi, quid observare me velis.

[Mehrere Personen haben mich gebeten, ihnen nach dem Vorbild der Prokonsuln zu gestatten, die Gebeine ihrer Angehörigen wegen der Schädigung durch das Alter des Grabes,

\footnotetext{
6 Etwas positiver urteilt Sherwin-White (1966: 547). Die Arbeit von Hoffer (1999) bietet eine Interpretation ausgewählter Briefe des ersten Buches, die diese unter dem Aspekt der Angst (anxiety) und ihrer Unterdrückung liest, vgl. S. 1: „The leading trait in Pliny's epistolary selfportrait is his confidence: confidence in himself and his friends, in their writings and activities, in the Roman government, and in the emperor [...] This very absence of anxiety invites us to look at the opposite side of the picture, at Pliny's anxieties, to help us understand his aims in putting together and publishing the letters.“
} 
wegen der Überschwemmungen des Flusses und aus allen möglichen andern Gründen umzubetten. Da ich weiß, daß man sich in unsrer Stadt bei derartigen Fragen an das Kollegium der Pontifices zu wenden pflegt, glaube ich, Dich, Herr, als Pontifex Maximus fragen zu müssen, wie ich mich verhalten soll. (Kasten 1968: 619)]

\subsection{TRAIANUS PLINIO}

Durum est iniungere necessitatem provincialibus pontificum adeundorum, si reliquias suorum propter aliquas iustas causas transferre ex loco in alium locum velint. Sequenda ergo potius tibi exempla sunt eorum, qui isti provinciae praefuerunt, et ut causa cuique, ita aut permittendum aut negandum.

[Es wäre hart, wollte man den Provinzialen den Zwang auferlegen, sich an die Pontifices zu wenden, wenn sie aus irgendwelchen triftigen Gründen die Gebeine ihrer Angehörigen von einem Ort an einen andern umbetten wollen. Du wirst Dich also besser an das Vorbild der früheren Statthalter Deiner Provinz halten und je nachdem, welche Gründe jeweils vorliegen, die Erlaubnis erteilen oder versagen. (Kasten 1968: 619)]

Ganz ähnlich verhält es sich mit den im Sommer des Jahres 110 n. Chr. verfassten Briefen 10.15-17a. In ihnen informiert Plinius den Kaiser ausführlich über seine Antrittsreise in die Provinz, hält ihn über jede Station in Kenntnis, einschließlich der aktuellen Wetterbedingungen und des persönlichen Gesundheitszustandes:

\subsection{PLINIUS TRAIANO IMPERATORI}

Quia confido, domine, ad curam tuam pertinere, nuntio tibi me Ephesum cum omnibus meis ن் $\dot{\rho} \rho \alpha \lambda \dot{\varepsilon} \alpha v$ navigasse quamvis contrariis ventis retentum. Nunc destino partim orariis navibus, partim vehiculis provinciam petere. Nam sicut itineri graves aestus, ita continuae navigationi etesiae reluctantur.

[Weil ich überzeugt bin, Herr, daß Du Wert darauf legst, melde ich Dir, daß ich mit allen meinen Leuten zu Schiff an Kap Malea vorbei, obwohl durch widrige Winde aufgehalten, nach Ephesus gelangt bin. Jetzt beabsichtigte ich, teils mit Küstenfahrzeugen, teils zu Wagen meine Provinz zu erreichen. Denn wie die drückende Hitze die Reise zu Lande erschwert, so die Passatwinde eine ununterbrochene Seefahrt. (Kasten 1968: 573)]

\subsection{TRAIANUS PLINIO}

Recte renuntiasti, mi Secunde carissime. Pertinet enim ad animum meum, quali itinere provinciam pervenias. Prudenter autem constituis interim navibus, interim vehiculis uti, prout loca suaserint.

[Es war recht, mein Secundus, daß Du mir Meldung gemacht hast; natürlich interessiert es mich, wie Deine Reise in die Provinz verläuft. Dein Entschluß, Dich je nach den Örtlichkeiten zeitweise der Schiffe, zeitweise der Wagen zu bedienen, ist vernünftig. (Kasten 1968: 573)] 


\subsection{7a C. PLINIUS TRAIANO IMPERATORI}

Sicut saluberrimam navigationem, domine, usque Ephesum expertus ita inde, postquam vehiculis iter facere coepi, gravissimis aestibus atque etiam febriculis vexatus Pergami substiti. Rursus, cum transissem in orarias nauculas, contrariis ventis retentus aliquanto tardius quam speraveram, id est XV Kal. Octobres, Bithyniam intravi. Non possum tamen de mora queri, cum mihi contigerit, quod erat auspicatissimum, natalem tuum in provincia celebrare. [...] Haec tibi, domine, in ipso ingressu meo scripsi.

[Herr, wenn mir die Seereise bis Ephesus sehr gut bekommen ist, so habe ich, seit ich im Wagen reise, sehr unter der drückenden Hitze und auch an leichten Fieberanfällen gelitten und deshalb in Pergamum haltgemacht. Als ich dann auf Küstenschiffe übergestiegen war, wurde ich wieder durch Gegenwinde festgehalten und gelangte so wesentlich später als erwartet, das heißt: erst am 17. September nach Bithynien. Trotzdem brauche ich die Verzögerung nicht zu bedauern, denn ich durfte - ein sehr gutes Omen! - Deinen Geburtstag bereits in der Provinz feiern. [...] Dies schreibe ich Dir, Herr, unmittelbar nach meinem Eintreffen. (Kasten 1968: 573)]

Die beiden Beispiele geben einen Einblick in Thematik und Stil der Korrespondenz zwischen Plinius und Trajan, deren Hauptkennzeichen das allgemein konstatierte Mitteilungs- und Absicherungsbedürfnis des Statthalters ist (oder zu sein scheint). Ist dies unsere moderne Reaktion auf vormoderne Praktiken von Herrschaftskommunikation oder mag der Kaiser ähnlich gedacht haben wie wir heute? Eindeutig beantworten lässt sich diese Frage nicht, doch könnte man geneigt sein, in Trajans Antwortschreiben epist.10.16 - „Es war recht, mein Secundus, daß Du mir Meldung gemacht hast; natürlich interessiert es mich, wie Deine Reise in die Provinz verläuft“ - einen Hauch von Ironie in der Stimme des Kaisers zu entdecken.

Aber ist dies schon das ganze Bild? Betrachtet man die Briefe etwas genauer und unterstellt ihnen ein Mehr an Literarizität als bisher zugestanden, wird man mit einem anderen Plinius konfrontiert, einem Plinius, der wesentlich weniger passiv und unsicher agiert, als es scheint. Einen ersten Hinweis liefert die Beobachtung, dass insbesondere die einleitenden Briefe des zehnten Buches in auffälliger Dichte eine Reihe von römischen sogenannten ,Wertbegriffen ‘ fallen lassen, die zum Kern von Trajans Selbstdarstellung als ,gutem Kaiser' gehören, darunter pietas, clementia und iustitia (vgl. Eck 2010: 116). Begriffe wie ,Frömmigkeit, Milde und Gerechtigkeit' sind dabei nicht auf Trajan beschränkt, sondern gehören zur Hochsemantik römischen Selbstverständnisses überhaupt und sind in der Kaiserzeit insbesondere mit Kaiser Augustus (63 v. Chr.-13 n. Chr.) verbunden, der in der Traditionsbildung schon früh zum idealen und vorbildlichen Kaiser par excellence avancierte (Galinsky 1996: 80-140; Noreña 2001; Christ 2009: 168-171). Die genannten Begriffe stellen Trajan aber nicht nur auf die Seite einer positiven Augustus-Tradition, sondern heben ihn zugleich von, schlechten 
Kaisern' wie Nero oder Domitian ab (Witschel 2010: 110). Domitian, 96 n. Chr. unter teilweise unklaren Umständen im eigenen Palast ermordet, verfiel sofort nach seinem Tod einer ,Erinnerungsverdammung (damnatio memoriae) und wurde zu einem der am meisten verhassten Kaiser, besonders unter Mitgliedern der senatorischen Elite, zu der auch Plinius und Tacitus gehörten. Auf Domitian folgte zunächst Marcus Cocceius Nerva (30-98 n. Chr.), der die Macht durch Adoption an Trajan übergab (Regierungszeit 98-117 n. Chr.). In seiner Korrespondenz macht sich Plinius dieses offizielle Werteschema strategisch zunutze, um Trajan lobend als ,guten Kaiser` zu charakterisieren, in deutlicher Abgrenzung zum ,schlechten Kaiser‘ Domitian, dessen als tyrannisch empfundener Regierungsstil vielen seiner Zeitgenossen noch lebendig vor Augen stand. Ein Beispiel für diese Technik findet sich bereits im ersten Brief des Buches epist. 10.1:

\subsection{PLINIUS TRAIANO IMPERATORI \\ [1] Tua quidem pietas, imperator sanctissime, optaverat, ut quam tardissime succederes patri; sed di immortales festinaverunt virtutes tuas ad gubernacula rei publicae quam susceperas admovere. Precor ergo ut tibi et per te generi humano prospera omnia, id est digna saeculo tuo contingant. Fortem te et hilarem, imperator optime, et privatim et publice opto.}

[In Deiner Sohnesliebe hattest Du, hocherhabener Kaiser, gewünscht, Deinem Vater so spät wie möglich zu folgen, aber die unsterblichen Götter haben es eilig gehabt, Deine Fähigkeit ans Ruder des Staates zu bringen, zu dessen Führung Du Dich bereit erklärt hattest. Ich bete also, daß Dir und durch Dich der ganzen Menschheit lauter Segen, und das heißt: alles, was Deiner Zeit würdig ist, beschieden sein möge. Gesundheit und Frohsinn wünsche ich Dir, bester Kaiser, für mich persönlich und im Namen des Staates. (Kasten 1968: 559)]

Mit der Wendung ,Deinem Vater so spät wie möglich zu folgen` bezieht sich Plinius auf den unerwarteten Tod Nervas Anfang 98 n. Chr., der den Weg für Trajans Herrschaft ebnete. Betrachtet man diesen Brief nicht isoliert, sondern im Buchkontext, verwundert es nicht, dass Plinius den Briefwechsel so eröffnet, dass das zentrale Thema ,Tugenden“ (virtutes) gleich zu Beginn Erwähnung findet (virtutes tuas - ,Deine Tugenden'). Im Lateinischen bezeichnet virtus zunächst allgemein „the qualities typical of a true man, manly spirit, resolution, valour, steadfastness or sim“. In einem engeren Sinne steht virtus für „excellence of character or mind, worth, merit, ability, etc.“ oder für „a particular excellence of character, ability, etc.“ (OLD 1988: 2073). Angesichts dieses lexikalischen Bedeutungsspektrums kann der Gebrauch von virtus an der vorliegenden Stelle als eine strategisch eingesetzte Polysemie (durch Spezialisierung) verstanden werden, mit der Plinius einerseits die allgemeinen Verdienste und Talente des neuen Kaisers lobend hervorhebt und sich zugleich einen Ausgangspunkt schafft, um im Weiteren diejenigen unter virtus subsumierten Wertbegriffe ins 
Spiel zu bringen, die für Trajans Selbstrepräsentation als Kaiser eine zentrale Rolle spielten. Im selben Brief kommt Plinius bereits auf eine besondere virtus zu sprechen, wenn er eingangs Trajans Frömmigkeit (pietas) hervorhebt, die ihn davon abhalte, die Macht in übermäßiger Eile $\mathrm{zu}$ suchen. Trajans Tugenden werden dann in den Briefen immer wieder thematisiert (z. B. epist. 10.14, 10.88, 10.100). In Brief 10.14 preist Plinius den Kaiser beispielsweise für einen Sieg gegen die Daker 112 n. Chr. mit den folgenden Worten:

10.14 C. PLINIUS TRAIANO IMPERATORI

Victoriae tuae, optime imperator, maximae, pulcherrimae, antiquissimae et tuo nomine et rei publicae gratulor, deosque immortales precor, ut omnes cogitationes tuas tam laetus sequatur eventus, cum virtutibus tantis gloria imperii et novetur et augeatur.

[Zu Deinem überwältigenden, herrlichen, bedeutsamen Siege beglückwünsche ich Dich, bester Kaiser, in Deinem und des Staates Namen und bitte die unsterblichen Götter, daß allen Deinen Unternehmungen ein ebenso glücklicher Erfolg beschieden sei, denn durch solche Heldentaten wächst und erneuert sich unsres Reiches Ruhm. (Kasten 1968: 571)]

\section{Wortspiele, at work}

Möchte man diese Analyse einen Schritt weiterführen, kann eine zweite Beobachtung hinzugefügt werden. Plinius lässt nicht nur einfach eine Reihe römischer Wertbegriffe und -konzepte fallen, die in Einklang mit Trajans Herrschaftsrepräsentation stehen. Vielmehr spielt er mit diesen Begriffen, indem er sie nicht einfach auf Trajan anwendet, sondern sie - auf subtile Weise und unter strategischer Nutzung lexikalischer Ambiguitäten - auch auf die eigene persona bezieht. Damit schafft er über den Weg der Sprache eine atmosphärische Nähe, die die politische Schwelle zwischen Kaiser und Senator geschmeidig überspannt. Ein markantes Beispiel für diese Technik findet sich in den Briefen acht und neun des zehnten Buches, in denen Plinius den Herrscher um Gewährung eines Urlaubes von 30 Tagen bittet. Die geplante Reise soll Plinius nach Tifernum Tiberinum (heute Città di Castello in Umbrien) führen, wo er einen Tempel mit einer Gruppe römischer Kaiserstatuen errichten möchte. Zu den vorhandenen Statuen soll aus eigenem Bemühen eine Statue des aktuellen Herrschers Trajan hinzugefügt werden. Der zweite Teil der Reise dient der Visitation von Ländereien, die sich im Besitz seiner Familie befinden und die im Jahr eine Rendite von mehr als 400.000 Sesterzen erwirtschaften (Seelentag 2004: 183-197). Dass die Reise öf- 
fentliche Pflicht (Tempelbau) und private Angelegenheiten (Vermögensverwaltung) verbindet, diskutiert der Senator offen in seinem Brief. Er schließt ihn mit den Worten:

\subsubsection{PLINIUS TRAIANO IMPERATORI}

Debebo ergo, domine, indulgentiae tuae et pietatis meae celeritatem et status ordinationem, si mihi ob utraque haec dederis commeatum XXX dierum. Neque enim angustius tempus praefinire possum, cum et municipium et agri de quibus loquor sint ultra centesimum et quinquagesimum lapidem.

[Also Deiner Huld, Herr, werde ich die alsbaldige Ausführung meines pietätvollen Wunsches und die Ordnung meiner Vermögensverhältnisse zu danken haben, wenn Du mir aus diesen beiden Gründen einen einmonatigen Urlaub bewilligst. Eine kürzere Frist kann ich nämlich nicht ansetzen, da das Städtchen und die Besitzungen, von denen ich spreche, jenseits des 150. Meilensteins liegen. (Kasten 1968: 567)]

Elegant verbindet Plinius hier Trajans ,Huld‘ (indulgentia tua) mit dem eigenen ,pietätsvollen Wunsch / Frömmigkeit‘ (pietas mea) in einem einzigen Satz. Cotton (1984) hat die zentrale Rolle herausgestellt, die indulgentia in Plinius' Haltung gegenüber dem Kaiser spielt und Seelentag (2004: 196) weist zurecht darauf hin, dass indulgentia und pietas an der vorliegenden Stelle eine klare Hierarchie zwischen Kaiser und Senator markieren. Was diese Partie aber besonders interessant macht, ist die Tatsache, dass der Begriff ,Frömmigkeit' (pietas) hier nicht zum ersten Mal innerhalb des Briefwechsels ins Spiel kommt. Denn schon im allerersten Brief lobt Plinius die Tugenden des Kaisers im Allgemeinen (virtutes tuas; epist. 10.1) und seine besondere Frömmigkeit im Besonderen (tua quidem pietas). Über die Technik der - auf den ersten Blick unauffälligen und geradezu natürlich wirkenden - Wortwiederholung gelingt es Plinius, das Thema ,Frömmigkeit' vom Kaiser (epist.1) auf sich selbst zu übertragen (epist. 8). Wie im Falle von virtus macht sich Plinius auch bei pietas lexikalische Polysemie zunutze. Denn im Lateinischen kann pietas „the relationship between human beings“ wie beispielsweise „of children to parents“ bezeichnen (in epist. 1: Trajans Verhältnis zu seinem Vorgänger und Adoptivvater Nerva). Zugleich wird es aber auch verwendet, wenn es um die Haltung „of citizens towards a State or ruler“ geht (epist. 8: Plinius' Verhältnis zu Trajan; vgl. OLD 1988: 1378). Genau in dieser zweiten Bedeutung verwendet Trajan pietas selbst in seinem Antwortschreiben:

\subsection{TRAIANUS PLINIO}

Et multas et omnes publicas causas petendi commeatus reddidisti; mihi autem vel sola voluntas tua suffecisset. Neque enim dubito te, ut primum potueris, ad tam districtum officium reversurum. Statuam poni mihi a te eo quo desideras loco, quamquam eius modi honorum parcissimus tamen patior, ne impedisse cursum erga me pietatis tuae videar. 
[Du führst viele persönliche und eine Reihe allgemeiner Gründe für Dein Urlaubsgesuch an; mir hätte allein Dein Wunsch genügt. Denn ich zweifle nicht, daß Du so bald wie möglich zu Deinem so arbeitsreichen Amte zurückkehren wirst. Damit, daß Du mir an dem in Aussicht genommenen Platze eine Statue errichten willst, bin ich, obwohl sonst sehr sparsam mit der Bewilligung derartiger Ehrungen, trotzdem einverstanden, um nicht den Anschein zu erwecken, als wollte ich die Äußerungen Deiner Ergebenheit gegen mich verhindern. (Kasten 1968: 569)]

Zwischen Brief eins und acht finden wir das Motiv der Frömmigkeit (pietas) noch einmal in Brief vier, wo diese einem gewissen Voconius Romanus zugeordnet wird, einem Bekannten des Plinius, der häufiger auch in den ersten neun Büchern des Gesamtkorpus vorkommt (Birley 2000: 101; Fein 1994: 104-105; Gibson und Morello 2012: 149-154). Plinius empfiehlt den Freund mit großer Emphase beim Kaiser und charakterisiert ihn als einen Mann, der durch exzellente Bildung und außerordentliche Frömmigkeit (pietas) hervorrage (,... non sine magna fiducia subsigno apud te fidem pro moribus Romani mei, quos et liberalia studia exornant et eximia pietas“ [... verbürge ich mich bei Dir nicht ohne große Zuversicht für die Persönlichkeit meines Romanus, den Vorliebe für die freien Künste und eine außergewöhnliche Anhänglichkeit auszeichnet], epist. 10.4, Üb. Kasten 1968: 563). Betrachtet man die Sequenz der ersten Briefe des zehnten Buches, so sieht man das zentrale Wort ,Frömmigkeit‘ unauffällig vom Kaiser über eine dritte Person (Voconius Romanus) zu Plinius selbst übergleiten. Diese Beobachtung ist umso interessanter, als pietas eines der wichtigsten römischen Wertekonzepte überhaupt darstellt, das aufs engste mit dem Idealbild römischer Herrschaft in der Augustus-Tradition zurückgeht (Galinsky 1996: 86-88; für Trajan s. Seelentag 2004: 65). Nicht zufällig ist pius auch die Haupteigenschaft des Helden Aeneas in Vergils Aeneis, einem Werk, das auf einer mythisch-historischen Verknüpfung der Aeneas-Figur mit Augustus beruht. In diesem im weiteren Sinne ,politischen' Gebrauch des Wortes steht der (kultische oder religiöse) Aspekt der Frömmigkeit gegenüber Ahnen und Göttern im Zentrum (vgl. OLD 1988: 1378, nr. 2).

Die Technik, die Sphäre des Kaisers durch Wortwiederholung mit der eigenen Person zu verknüpfen, ist nicht auf ein Beispiel beschränkt. Wiederum im ersten Brief des zehnten Buches finden wir eine funktional vergleichbare Wiederholung des Verbs optare (,wünschen'). Im ersten Fall bezieht sich optare auf Trajan (,In Deiner Sohnesliebe hattest Du [...] gewünscht“), im zweiten auf Plinius („Gesundheit und Frohsinn wünsche ich Dir [...]“). Was auf den ersten Blick unauffällig erscheint, gewinnt im Kontext der oben beschriebenen Wortwiederholungstechnik an Bedeutung. Wieder wird ein Wort zunächst im Zusammenhang mit Trajan und dann mit Bezug auf Plinius selbst verwendet, diesmal sogar nicht über Briefgrenzen hinweg, sondern innerhalb ein und desselben kurzen 
Briefes. Die zitierten Beispiele erzeugen eine Einheit oder Gleichklang zwischen Kaiser und Senator, im Fall von pietas über gemeinsame Werte und im Falle von optare über den Freundschaftsgedanken gemeinsamen Strebens. Letzteres erinnert an die in der antiken Tradition geläufige Definition von amicitia als ein ,dasselbe wünschen und dasselbe nicht wünschen'. So schreibt der römische Historiker Sallust: nam idem velle atque idem nolle, ea demum firma amicitia est (Sallust, Catilina 20.3). ${ }^{7}$

Die bisher beschriebenen Wortspiele beruhen im Kern auf kontextueller Integration und machen sich zugleich Eigenschaften der sprachlichen Einheiten wie Polysemie zunutze (Definitionen bei Winter-Froemel 2009 und 2016; Zirker und Winter-Froemel 2015b: 5 mit weiterer Lit.). Das Hauptmerkmal ist die Wiederholung derselben lexikalischen Einheit in verschiedenen Zusammenhängen, um zwei gegebene Textelemente miteinander zu verknüpfen (Winter-Froemel 2016: 18), in unserem Fall Trajan und Plinius. Nicht unwichtig, aber sekundär ist die Frage, ob die Wiederholung eine Bedeutungsverschiebung bewirkt, wie im Fall von pietas, einem Wort, das auf ganz verschiedene Arten sozialer Relationen angewendet werden kann und das je nach Kontext von Beziehungen zwischen gleichrangigen Personen zu solchen Verhältnissen reicht, in denen eine Seite über oder unter der anderen steht. Um diese Verschiebung der lexikalischen Bedeutung auszudrücken, übersetzt Kasten pietas in epist. 1 in Bezug auf Trajan mit ,Sohnesliebe‘ (d. h. zu seinem Vorgänger Nerva) und später in epist. 8 und 9 in Bezug auf Plinius mit ,pietätsvoller Wunsch' (d. h. zu seinem Kaiser Trajan).

Wortspiele können in der Alltagskommunikation und in literarischen Texten unterschiedliche Funktionen erfüllen. Oft haben sie eine unterhaltsame oder komische Wirkung, manchmal werden sie verwendet, um eine Figur zu charakterisieren oder die Aufmerksamkeit des internen oder externen Lesers oder Hörers zu erregen. Wortspiele können auch der Strategie eines Redners dienen „to present him-/ herself in a positive, creative way“ (Zirker und WinterFroemel 2015b: 7). Diese Funktion des self-fashioning bietet eine Kategorie, die der kommunikativen Situation in Plinius' zehntem Buch entspricht. Die Wortspiele schaffen eine Einheit zwischen dem Kaiser und dem Senator mit dem Effekt, dass beide Seiten, trotz aller verbleibender Unterschiede in Rang und Macht, fast gleichwertig und nahezu als Freunde erscheinen. Die spielerische Sprachmanipulation dient dann auch der Manipulation der sozialen Ordnung. Aber wie weit reicht diese Manipulation? Betrifft sie nur den internen Leser und Dialogpartner Trajan oder reicht es darüber hinaus bis zum externen Leser einer

7 Zum Thema Freundschaft bei Plinius s. Gibson und Morello (2012: 347 s. v. friendship), Konstan (1997: 147-148), Castagna (2003). 
erst später angefertigten und veröffentlichten Briefsammlung? Über die Absichten und Strategien des Autors zu sprechen, ist bei antiken Schriftstellern besonders schwierig, über die Hintergründe des zehnten Briefbuches ist so gut wie nichts bekannt. Dennoch findet sich an anderer Stelle im Werk des Plinius ein signifikanter Beleg für die hier postulierte bewusste Strategie, eine Nah- oder Freundschaftsbeziehung zwischen Kaiser und Senatorenstand zu etablieren. Am 1. September $100 \mathrm{n}$. Chr. hielt Plinius eine feierliche Rede im Senat und in Anwesenheit des Kaisers. Dieser erhaltene sogenannte Panegyricus Traiani erlangte in der Antike schnell große Bekanntheit und avancierte in der Spätantike zum Vorbild vergleichbarer Lobreden auf den Kaiser (Rees 2011). In dieser Rede preist Plinius nicht nur Trajan als ,besten Herrscher (optimus princeps), sondern bezieht sich unmittelbar auf die Freundschaft, die Trajan den Senatoren anbiete, die unter der Herrschaft des ,schlechten Herrscher' (Domitian) gelitten hätten (Roche 2011b: 18-22). Gegen Ende seiner Rede hebt Plinius das Freundschaftsmotiv noch einmal mit großer Emphase hervor (Noreña 2011: 31-32):

[85] Iam etiam et in privatorum animis exoleverat priscum mortalium bonum amicitia, cuius in locum migraverant adsentationes blanditiae et peior odio amoris simulatio. Etenim in principum domo nomen tantum amicitiae, inane scilicet inrisumque manebat. [...] Tu hanc pulsam et errantem reduxisti: habes amicos quia amicus ipse es. [...] Diligis ergo cum diligaris, et in eo quod utrimque honestissimum est, tota gloria tua est; qui superior factus, descendis in omnia familiaritatis officia, et in amicum ex imperatore submitteris, immo tunc maxime imperator cum amicum agis.

[Ein altehrwürdiges Gut der Menschen, die Freundschaft, war längst in den Herzen auch der einfachen Bürger verkümmert, und an seine Stelle traten Anbiederung, Schmeichelei und, schlimmer noch als Haß, geheuchelte Liebe. Ja, im Kaiserpalast blieb nur das Wort ,Freundschaft' noch übrig, ein leerer, verspotteter Begriff, sonst nichts. [...] Du warst es, der diese vertriebene, heimatlos umherirrende Freundschaft wieder zurückgeführt hat; du nennst gute Freunde dein eigen, weil du selbst ihnen ein guter Freund bist. [...] Da du also geliebt wirst, liebst du wieder - ein Verhältnis, das beiden Seiten hohe Ehre macht, Ruhm aber ganz allein dir bringt. Denn du stehst höher und lässest dich dennoch herab, alle Pflichten der Freundschaft zu erfüllen, legst den Kaiser ab und wirst schlicht nur ein Freunddoch nein, gerade dann bist du am meisten Kaiser, wenn du dich als Freund gibst. (Kühn 2008: 164-167)]

Es darf als wahrscheinlich gelten, dass Plinius das hier formulierte Ideal der Freundschaft im pragmatischen Kontext seines Briefwechsels mit dem Kaiser fortsetzt. ${ }^{8}$ Es ist auch wahrscheinlich, dass er dasselbe Publikum im Auge hat wie

8 Vgl. Konstan (1997: 148) mit Bezug auf einen früheren Passus des Panegyricus, in dem Plinius ebenfalls über Freundschaft spricht: „[...] Pliny also implies (44.7) that friendship with Trajan 
beim Anlass des Panegyricus, nämlich Kaiser Trajan selbst und zugleich seine römischen Mit-Senatoren.

\title{
5 Kaiser Trajan als ,Model Reader
}

Abschließend können noch zwei Aspekte hinzugefügt werden. Die Art des hier besprochenen Wortspiels verlangt vom Leser eine sorgfältige und genaue Lektüre des Textes. Einige Merkmale treten nur durch Prozesse von Zweitlektüre (rereading) und ständiges Hin- und Herblättern hervor. Dieses Phänomen von (intentionalen) Wortspielen, die nicht auf direktes Verstehen abzielen, hat Bauer (2015) als „secret wordplay“ beschrieben:

\begin{abstract}
Wordplay frequently aims at effects that go along with a certain processing effort: the reader or hearer must notice that there is a play on words intended, must realize its meaning, and will, as a rule, take pleasure in the discovery. Wordplay thus establishes a bond between speaker and hearer: the speaker assumes that the hearer will be able to get it and thus pays his audience a compliment, which is returned by their appreciation and expression of delight. Authors, however, may wish to heighten the pleasure by deferring it, or they may have other reasons for raising the hurdles, turning the play on words into a mystery that is only to be solved by a select, knowledgeable audience. (Bauer 2015: 269-270)
\end{abstract}

Bauers Beobachtung, dass geheime Wortspiele vor allem in literarischen Texten zuhause sind (2015: 270), bietet darüber hinaus ein Argument für die Diskussion über die literarische Eigenart des zehnten Buches und ihren Bezug zu den ersten neun Büchern. Denn wenn man solche ,geheimen Wortspiele‘ in der offiziellen Korrespondenz zwischen Plinius und Trajan beobachten kann, sagt dies auch etwas über den im Text konstruierten Modell-Leser aus. Es handelt sich um einen Leser, der derartige Wortspiele zu entdecken vermag, der mit derartigen Techniken rechnet und die dadurch ausgesprochene, invitation to metalinguistic reflection“ akzeptiert (Zirker und Winter-Froemel 2015b: 10). In diesem Sinne erzeugt Plinius nicht nur eine Atmosphäre der Verbundenheit und Freundschaft mit Trajan, sondern er macht - indem er seinen Briefen eine spielerische Dimension hinzufügt - auch den Kaiser zu einem ,Model Reader‘. Und dies sagt uns schließlich auch etwas Entscheidendes über Plinius selbst: Als ,literarischer Wortspieler' präsentiert er sich auch in Buch 10 als ein weit weniger unsicherer und schwacher Mensch als bisher angenommen, die Machtverhältnisse zwischen ihm und

depends on the moral equality between the emperor and those who are, by reason of their virtue, similar (similes) to him.“ 
dem Kaiser zeigen sich nicht grundlegend gewandelt, aber doch anders und vielschichtiger disponiert.

Ähnlich wie das ,animistische Potential' des Bleistifts bei Yōko Tawada durch die Brechung einer Mehrsprachenperspektive hervortritt, gewinnt Plinius' Strategie an Kontur, wenn seine Briefe an den Kaiser als ein Text wahrgenommen werden, der auf der Schnittstelle zwischen Alltagskommunikation und Literatur liegend sich Ausdrucksmöglichkeiten zunutze macht, die man in einer Verwaltungskorrespondenz zunächst nicht erwartet.

\section{Literaturangaben}

\section{Zitierte Primärtexte}

Glare, Peter G. W. 2012. Oxford Latin Dictionary. Second Edition. 2 Vol. Oxford: Oxford University Press. [= OLD]

Kasten, Helmut. 1968. C. Plini Caecilii Secundi Epistularum libri decem. Gaius Plinius Caecilius Secundus, Briefe. Lateinisch-deutsch. München: Heimeran.

Kühn, Werner. ${ }^{2} 2008$. Panegyrikus. Lobrede auf den Kaiser Trajan/Plinius der Jüngere. Herausgegeben, eingeleitet und übersetzt von Werner Kühn, Darmstadt: Wissenschaftliche Buchgesellschaft.

Mynors, Roger A. B. 1963. C. Plini Caecili Secundi epistularum libri decem. Recognovit brevique adnotatione critica instruxit. Oxford: Oxford University Press.

Mynors, Roger A. B. 1964. XII Panegyrici Latini. Oxford: Clarendon Press.

Tawada, Yōko. 1996. Talisman. Literarische Essays. Tübingen: Konkursbuchverlag Claudia Gehrke.

\section{Weitere zitierte Literatur}

Ahl, Frederick. 1985. Metaformations. Soundplay and Wordplay in Ovid and other Classical Poets. Ithaca: Cornell University Press.

Barchiesi, Alessandro. 2005. The Search for the Perfect Book. A PS to the New Posidippus. In Kathryn Gutzwiller (Hg.), The New Posidippus. A Hellenistic Poetry Book, 320-340. Oxford: Oxford University Press.

Bauer, Matthias 2015. Secret Wordplay and What It May Tell Us. In Angelika Zirker \& Esme Winter-Froemel (Hgg.), Wordplay and Metalinguistic/Metadiscursive Reflection. Authors, Contexts, Techniques, and Meta-Reflection (The Dynamics of Wordplay 1), 269-288. Berlin \& Boston: De Gruyter.

Birley, Anthony R. 2000. Onomasticon to the Younger Pliny. Letters and Panegyric. Berlin: De Gruyter.

Bracci, Francesco. 2011. Plinio il Giovane. Epistole, Libro X. Introduzione, traduzione e commento. Pisa: Pisa University Press. 
Castagna, Luigi. 2003. Teoria e prassi dell'amicizia in Plinio il Giovane. In Luigi Castagna \& Eckard Lefèvre (Hgg.), Plinius der Jüngere und seine Zeit, 145-172. München: Saur.

Castagna, Luigi \& Eckard Lefèvre (Hgg.). 2003. Plinius der Jüngere und seine Zeit. München: Saur.

Christ, Karl. 2009. Geschichte der römischen Kaiserzeit. Von Augustus bis Konstantin. 6. Auflage. München: C. H. Beck.

Cotton, Hannah. 1984. The Concept of indulgentia under Trajan. Chiron 14. 245-266.

Eck, Werner. 2010. Trajan. 98-117. In Manfred Clauss (Hg.), Die römischen Kaiser. 4., aktualisierte Auflage, 110-124. München: C. H. Beck.

Eco, Umberto. 1985. A Portrait of the Elder as a Young Pliny. How to build Fame. In Marshall Blonsky (Hg.), On Signs, 289-302. Oxford: Blackwell.

Fein, Sylvia. 1994. Die Beziehungen der Kaiser Trajan und Hadrian zu den ,litterati‘. Stuttgart: Teubner.

Galinsky, Karl. 1996. Augustan Culture. An Interpretative Introduction. Princeton / Chichester: Princeton University Press.

Gibson, Roy K. \& Ruth Morello. 2012. Reading the Letters of Pliny the Younger. An Introduction. Cambridge: Cambridge University Press.

Gibson, Roy K. \& Christopher Whitton (Hgg.). 2016. The Epistles of Pliny. Oxford: Oxford University Press.

Henderson, John. 2002. Pliny's Statue. The Letters, Self-Portraiture and Classical Art. Exeter: University of Exeter Press.

Hoffer, Stanley E. 1999. The Anxieties of Pliny the Younger. Atlanta: Scholars Press.

Konstan, David. 1997. Friendship in the Classical World. Cambridge: Cambridge University Press.

Ludolph, Matthias. 1997. Epistolographie und Selbstdarstellung. Untersuchungen zu den ,Paradebriefen' Plinius des Jüngeren. Tübingen: Narr.

Marchesi, Ilaria. 2008. The Art of Pliny's Letters. A Poetics of Allusion in the Private Correspondence. Cambridge: Cambridge University Press.

Marchesi, Ilaria (Hg.). 2015. Pliny the Book-Maker. Betting on Posterity in the Epistles. Oxford: Oxford University Press.

Millar, Fergus. 2016a. Imperial Letters in Latin. Pliny and Trajan, Egnatius Taurinus and Hadrian. Scripta Classica Israelica 35. 65-83.

Millar, Fergus. 2016b. Trajan. Government by Correspondence. In Roy K. Gibson \& Christopher Whitton (Hgg.), The Epistles of Pliny, 419-441. Oxford: Oxford University Press.

Mitsis, Philip \& Ioannis Ziogas (Hgg.). 2016. Wordplay and Powerplay in Latin Poetry. Berlin: De Gruyter.

Noreña, Carlos F. 2001. The Communication of the Emperor's Virtues. The Journal of Roman Studies 91. 146-168.

Noreña, Carlos F. 2007. The Social Economy of Pliny's Correspondence with Trajan. American Journal of Philology 128. 239-277.

Noreña, Carlos F. 2011. Self-Fashioning in the Panegyricus. In Paul Roche (Hg.), Pliny's Praise. Panegyricus in the Roman World, 29-44. Cambridge: Cambridge University Press.

O'Hara, James J. 1996. True Names. Vergil and the Alexandrian Tradition of Etymological Wordplay. Ann Arbor: The University of Michigan Press.

Rees, Roger. 2011. Afterwords of Praise. In Paul Roche (Hg.), Pliny's Praise. The Panegyricus in the Roman World, 175-188. Cambridge: Cambridge University Press. 
Roche, Paul. 2011a. (Hg). Pliny's Praise. The Panegyricus in the Roman World. Cambridge: Cambridge University Press.

Roche, Paul. 2011b. Pliny's Thanksgiving. An Introduction to the Panegyricus. In Paul Roche (Hg.), Pliny's Praise. The Panegyricus in the Roman World, 1-28. Cambridge: Cambridge University Press.

Seelentag, Gunnar. 2004. Taten und Tugenden Traians. Herrschaftsdarstellung im Principat. Stuttgart: Franz Steiner Verlag.

Sherwin-White, Adrian N. 1966. The Letters of Pliny. A Historical and Social Commentary. Oxford: Clarendon Press.

Soverini, Paolo. 1989. Impero e imperatori nell'opera di Plinio il Giovane. Aspetti e problemi del rapporto con Domiziano e Traiano. Aufstieg und Niedergang der römischen Welt, II 33.1, 515-554. Berlin: De Gruyter.

Stadter, Philip A. 2006. Pliny and the Ideology of Empire. The Correspondence with Trajan. Prometheus 32. 61-76.

Syme, Ronald. 1985. Correspondents of Pliny. Historia. Zeitschrift für Alte Geschichte 34. 324359.

Williams, Wynne. 1990. Correspondence with Trajan from Bithynia (Epistles X). Translated, with Introduction and Commentary. Warminster: Aris \& Phillips.

Winter-Froemel, Esme. 2009. Wortspiel. In Gert Ueding (Hg.), Historisches Wörterbuch der Rhetorik, Bd. 9, 1429-1443. Tübingen: Niemeyer.

Winter-Froemel, Esme. 2016. Approaching Wordplay. In Sebastian Knospe, Alexander Onysko, \& Maik Goth (Hgg.), Crossing Languages to Play with Words. Multidisciplinary Perspectives (The Dynamics of Wordplay 3), 11-46. Berlin \& Boston: De Gruyter.

Witschel, Christian. 2010. Trajan. 81-96. In Manfred Clauss (Hg.), Die römischen Kaiser. 4., aktualisierte Auflage, 98-110. München: C. H. Beck.

Woolf, Greg 2015. Pliny / Trajan and the Poetics of Empire. Classical Philology 110. 132-151.

Woolf, Greg 2016. Pliny's Province. In: Roy K. Gibson \& Christopher Whitton (Hgg.), The Epistles of Pliny, 422-460. Oxford: Oxford University Press.

Zirker, Angelika \& Esme Winter-Froemel (Hgg.). 2015a. Wordplay and Metalinguistic/Metadiscursive Reflection. Authors, Contexts, Techniques, and Meta-Reflection (The Dynamics of Wordplay 1). Berlin \& Boston: De Gruyter.

Zirker, Angelika \& Esme Winter-Froemel. 2015b. Wordplay and Its Interfaces in Speaker-Hearer Interaction: An Introduction. In Angelika Zirker \& Esme Winter-Froemel (Hgg.), Wordplay and Metalinguistic/Metadiscursive Reflection. Authors, Contexts, Techniques, and MetaReflection (The Dynamics of Wordplay 1), 1-22. Berlin \& Boston: De Gruyter.

Prof. Dr. Robert Kirstein (Tübingen) lehrt Lateinische Philologie am Philologischen Seminar der Eberhard Karls Universität Tübingen. Er ist u. a. Mitglied im Graduiertenkolleg 1808 „Ambiguität. Produktion und Rezeption“. Studium der Klassischen Philologie in Bonn, Oxford und Münster. Promotion mit einer Arbeit zum spätantiken Dichter Paulinus von Nola, Habilitation zur Bukolik in der Hellenistischen Literatur. Forschungsschwerpunkte: Dichtung der Hellenistischen und Augusteischen Zeit, Ovid, Narratologie, Raum in der Literatur, Ambiguität, Geschichte der Klassischen Philologie im 19. und 20. Jahrhundert. 